\title{
Review: behavioural treatment has a small to moderate beneficial effect on chronic low back pain
}

van Tulder MW, Ostelo R, Vlaeyen JW, et al. Behavioural treatment for chronic low back pain. Cochrane Database Syst Rev 2000;(1):CD002014 (latest version 15 Feb 2000).

\section{QUESTION: In patients with chronic non-specific low back pain, is behavioural treatment more effective than other treatments or placebo?}

\section{Data sources}

Studies were identified by searching 3 databases, scanning references of relevant studies and reviews, screening the Cochrane Library, and tracking citations of relevant randomised controlled trials (RCTs) in Science Citation Index.

\section{Study selection}

Studies were selected if they were RCTs of patients aged 18-65 years of age with non-specific chronic low back pain (symptoms persisting for $\geqslant 12$ wks), $\geqslant 1$ behavioural treatment was used, and $\geqslant 1$ important outcome was assessed. Outcomes were grouped into the following domains: behavioural outcomes, overall improvement, back pain specific functional status, generic functional status, return to work, and self reported pain intensity.

\section{Data extraction}

2 reviewers independently assessed the methodological quality of the trials (blinding and withdrawals), and extracted data on outcome domains. Effect sizes (ESs) were calculated for domains within each study for each comparison. ESs were pooled for each domain using a random effects model.

\section{Main results}

20 RCTs were included. 5 RCTs had $\geqslant 6$ positive quality assessment scores. 11 RCTs compared behavioural treatment with no treatment, waiting list controls, or placebo; 7 RCTs had data that could be pooled. Behavioural treatment had a moderate positive effect on pain intensity and a small positive effect on behavioural outcomes (table). 6 trials that added a behavioural component to such usual treatments as physiotherapy and back education, inpatient pain management, pharmacotherapy, and exercise treatment showed no short or long term effects of behavioural treatment on pain intensity, generic functional status, or behavioural outcomes (table). Differing types of behavioural treatment (cognitive, operant, respondent, and combinations) did not differ in effectiveness.

\section{Conclusion}

In patients with chronic non-specific low back pain, behavioural treatment has small to moderate beneficial effects on pain, functional, and behavioural outcomes.
Source of funding: Health Insurance Board of the Netherlands

For correspondence: Dr $M W$ van Tulder, Institute for Research in Extramural Medicine, Free University, van der Boechorststraat 7 1081 BT Amsterdam, the Netherlands. Fax +31204448181 .

\section{COMMENTARY}

Systematic reviews of physical treatments from the Cochrane Back Review Group have made a significant contribution to the pain field. But applying their methods to psychological treatments for pain produces problems that diminish the clinical usefulness of findings, although most are thoughtfully addressed in the discussion of this review by van Tulder et al, where the contribution of the 3 eminent psychologist co-authors is evident.

Despite an excellent search strategy and explicit selection criteria, 2 problems reduced the applicability of the results. Firstly, the review included only low back pain, which has no unique psychological significance. Secondly, Cochrane approved methodological quality ratings for physical or medical treatments were inadequately modified. A few were inappropriate (eg, blinding of care providers, as acknowledged in the discussion). Overall, the criteria are inadequate to describe psychological treatment trials and thereby misrepresent their quality. Although the authors recognised that shortcomings might lie in publication rather than study quality, the qualitative approach was used to grade the strength of evidence.

Use of effect sizes with CIs is well suited to these data, but clinical meaning is sacrificed by combining into a single treatment method all behavioural and cognitive treatments, and into a single "behavioural" outcome all behavioural, cognitive, and emotional data. Outcomes of pain and functional status are better distinguished and of clinical utility. Although they are derived from diverse treatment methods, these results can be treated with reasonable confidence. Overall, the review does not add substantially to the systematic review and meta-analysis of Morley et $a l,{ }^{1}$ nor should it discourage clinical use of psychological treatment methods in chronic pain.

Consensus on good psychological treatment trial design and reporting in the pain field will draw on the best of psychological treatment trials as well as on Cochrane conventions, including such variables as patient expectations of treatment, and care providers' expectations, skills, and adherence to trial protocol.

Ruth Allen, DClinPsy Amanda C de C Williams, PhD, CPsychol St Thomas'Hospital, London, UK

1 Morley SJ, Eccleston C, Williams A. Systematic review and meta-analysis of randomized controlled trials of cognitive behavior therapy and behavior therapy for chronic pain in adults, excluding headache. Pain 1999;80:1-13.

Behavioural treatments $v$ no treatment or other treatments for chronic low back pain

\begin{tabular}{|c|c|c|c|c|}
\hline Comparison & $\begin{array}{l}\text { Number } \\
\text { of trials }\end{array}$ & Outcomes at post-treatment & $\begin{array}{l}\text { Pooled effect size (ES) } \\
(95 \% \mathrm{CI})\end{array}$ & $\begin{array}{l}\text { Controls with a worse } \\
\text { outcome than treated }(\mathrm{Cl})^{*}\end{array}$ \\
\hline \multirow[t]{3}{*}{$\begin{array}{l}\text { Behavioural treatment } v \text { no treatment, waiting list } \\
\text { control, or placebo }\end{array}$} & 7 & Pain intensity & $0.62(0.25$ to 0.98$)$ & $73 \%$ (60 to 84$)$ \\
\hline & & Generic functional status & $0.35(-0.04$ to 0.74$)$ & $64 \%$ (48 to 77 ) \\
\hline & & Behavioural outcomes & $0.40(0.10$ to 0.70$)$ & $64 \%$ (54 to 76 ) \\
\hline \multirow[t]{8}{*}{$\begin{array}{l}\text { Behavioural treatment and another treatment } v \\
\text { other treatment alone }\end{array}$} & 6 & & & \\
\hline & & Pain intensity & $0.03(-0.30$ to 0.36$)$ & $51 \%(38$ to 64$)$ \\
\hline & & Generic functional status & $0.31(-0.01$ to 0.64$)$ & $62 \%$ (50 to 74 ) \\
\hline & & Behavioural outcomes & $0.19(-0.08$ to 0.45$)$ & $58 \%$ (47 to 67$)$ \\
\hline & & Outcomes at $\geqslant 6$ months & & \\
\hline & & Pain intensity & $-0.24(-0.64$ to 0.16$)$ & $41 \%$ (26 to 56$)$ \\
\hline & & Generic functional status & 0.26 (-0.06 to 0.57$)$ & $60 \%$ (48 to 72 ) \\
\hline & & Behavioural outcomes & $0.32(-0.06$ to 0.71$)$ & $63 \%(48$ to 76$)$ \\
\hline
\end{tabular}

*See glossary; calculated from data in article. 